\title{
INFLUENCE OF DISCOPLASTY AND DISCECTOMY OF THE TEMPOROMANDIBULAR JOINT ON ELIMINATION OF PAIN AND RESTRICTED MOUTH OPENING
}

\author{
Jiři Krug ${ }^{1}$, Zdeněk Jirousek ${ }^{1}$, Helena Šuchmová ${ }^{2}$ Eva Črmáková ${ }^{3}$ \\ Charles University in Prague, Faculty of Medicine in Hradec Králové, Czech Republic: Department of Dentistry ${ }^{1}$, \\ Computer Technology Center ${ }^{3}$; Hospital Liberec, Czech Republic: Department of Oral and Maxillofacial Surgery ${ }^{2}$
}

\begin{abstract}
Summary: The retrospective study was based on 36 open joint surgeries done in 33 consecutive patients with anterior disc dislocation without reduction with severe signs and symptoms and unsuccessful nonsurgical treatment lasting at least 6 months. The patients underwent either discoplasty or discectomy followed by auricular cartilage graft implantation and were evaluated for amount of interincisal mouth opening, severity of pain on VAS scores preoperatively and as well as 12 months postsurgically. The minimum pain levels were preoperatively 50 on VAS for the discoplasty group and 60 on VAS for the discectomy group. After the surgery, the VAS values lower than 20 were reported on $69.4 \%$ of 25 sides $(72.7 \%$ in the discoplasty group, $68.0 \%$ in the discectomy group). Using the cutoff point for maximal interincisal opening of more than $35 \mathrm{~mm}$, only 6 patients $(16.6 \%)$ fulfilled the criterion at the baseline. Interincisal distance measured vertically 12 months after the operation increased in all but five patients. In three of the five subjects, the opening was at the same level as before the surgery. Almost ninety-one percent of the patients (90.9\%) in discoplasty group and $81.8 \%$ in discectomy group, respectively reached $35 \mathrm{~mm}$ or more of vertical opening after the operation. Applying the 1984 Criteria for classification of postoperative results (AAOMS), 12 months after surgery, 24 patients $(72.7 \%)$ displayed good results, 6 patients ( $18.2 \%)$ were evaluated with acceptable results, and in 3 cases $(9.1 \%)$ we observed bad results. Although only short-term evaluation is presented, our findings show that both discoplasty and discectomy are effective methods for surgical treatment of patients suffering from severe temporomandibular pain and limited mouth opening. Discoplasty should be reserved only for patients without severe changes of the disc.
\end{abstract}

Key words: Discectomy; Discoplasty; Temporomandibular joint disorders; Autogenous cartilage graft

\section{Introduction}

Temporomandibular joint disorders (TMJD) represent a significant source of facial pain and mandibular dysfunction. More than $50 \%$ of the population exhibits symptoms of TMJD (10) and up to $30 \%$ of these people are in need of stomatognathic therapy $(2,21)$. Most of TMJD patients can be successfully treated by conservative methods. The patients who experience of repeatable severe symptoms of the TMJD not reflecting on conservative therapy and presenting with advanced joint pathology are indicated for open surgery (7). These modalities range from a minimally invasive technique such as an arthroscopy to a total joint replacement. The percentage of TMJD patients who need surgical intervention ranges from $1 \%$ to $25 \%(9,11,24)$. Currently, there are basically two commonly accepted surgical techniques. Discoplasty (discorhaphy, discopexis) typically involves a limited wedge resection of the retrodiscal tissue followed by disc repositioning. The operation may be combined with concomitant hard-tissue surgery (arthroplasty) on the articular eminence (eminectomy) or on the condyle (condylotomy). It seems that a morphologically normal-appearing dislocated disc can be surgically reduced to its anatomic position and maintained via posterior plication procedures. Several studies have reported on the success of this repositioning technique collectively suggesting an $80 \%$ to $96 \%$ success rate $(1,25)$. The timing of the follow-up ranged from 2 to 4 years. A successful patient outcome was generally defined as a decrease in pain and an increase in the range of mandibular movement, following surgery. There are occasions, however, when the disc is perforated, deformed, nonreducible, or otherwise not amenable to repair and we are more likely to remove it (20). Some studies with long-term follow-up show fair results in terms of pain relief and improving range of motion in patients having undergone discectomy without the disc replacement (8). In most instances, though, these patients eventually showed evidence of degenerative joint disease, clinically 
reflected by an increased frequency of crepitation, and in several patients developed fibrous adhesions, which limited mandibular movement (12). Some clinicians believe that interposition of some type of material between the articular surfaces is necessary to minimize the possibility of arthritic degeneration of joint surfaces and occlusal disturbances (17). Among autogenous materials, auricular cartilage has become attractive as a transplant in a temporomandibular joint (TMJ). Reconstructive arthroplasty has been shown to be a stage specific operation with excellent results in the stage II and good results in the stage III of the internal derangement of the TMJ, while discectomy is successful in cases of the stages IV (14) based on the Schellhas classification (23). The purpose of the study was to figure out the impact of both the disc repositioning and disc excision with auricular cartilage grafting replacement on patient treatment outcome parameters. We compared the decrease in pain and increase in range of motion after the two operating technique.

\section{Materials and Methods}

\section{Patient Selection and Preoperative Management}

The study was based on 36 open joint surgeries done in 33 consecutive patients with a range of 17 to 63 years $(37.1 \pm$ 14.2), during 4 years between March, 1998 and March, 2002. Ninety-seven percent of the subjects ( 32 of 33) were females. We indicated 11 patients (11 joints) aged from 26 to 46 years $(27.6 \pm 7.7)$ for discoplasty, (discoplasty group). Discectomy followed by auricular cartilage grafting was performed in 22 patients ( 25 joints) with a range of 17 to 63 years (41.1 \pm 15.0$)$, (discectomy group). In this group, three patients were operated on bilaterally. Prior to the surgery, all patients received a combination of counseling, occlusal bite plate therapy and adjustment, non-steroidal anti-inflammatory agents, arthrocentesis and/or simple self-administered physical therapy (thermal applications, range of motion and isometric exercises). We focused on eradication of muscular painful problems not to dissimulate the TMJ pain. Three patients underwent arthroscopy without benefit before the operation. Preoperative imaging was performed in all subjects to substantiate the presence of intra-articular disorders as suggested by clinical sings and symptoms. An advanced anterior disc displacement without reduction was confirmed on magnetic resonance imaging (MRI) in all 36 joints (Fig 1, 2). To be considered a candidate for TMJ surgery, all patients had to fulfill the same criteria. First, the nonsurgical treatment of the symptoms lasting at least 6 months before indicating an operation had to be deemed unsuccessful by the subjects, with the residual intolerable pain and dysfunction, especially during vertical mouth opening. Only the patients suffering from continuing problems were finally operated on. Second, the intra-articular pathology had to be documented by presurgical joint imaging and judged to be the source of the residual pain and/or dysfunction by the clinicians. Indications for the discecto- my included findings of disc perforation, gross deformity, and discs not thought to be amenable to stable posterior plication. In other situation, reposition of the disc was indicated. All patients were thoroughly examined before the surgery and at follow-up 12 months after surgical treatment with a special accent on the presence of pain and range of motion (ROM) $(5,21)$. Pain in the TMJ during mandibular function (opening of the mouth, biting, and chewing) was noticed using a $100-\mathrm{mm}$ visual analog scale (VAS) $(5,21)$. If there was no pain, it was recorded zero. Symmetrical mouth opening was recorded in millimeters during the pre- and postoperative periods. The distance between maxillary and mandibular central incisor cusp tips was measured without allowing for overbite. In the patients, where we performed the operation bilaterally, we counted only one value of ROM for both sides, however there were two VAS values from each side. Patients who were operated on more than once were excluded from the study. The postoperative results were principally classified according to the criteria described by the American Association of Oral and Maxillofacial Surgeons (AAOMS) (5) and modified by Eriksson et al. (8). Pain relief after surgery was considered satisfactory if the VAS score was less than 30. Mouth opening less than 35 $\mathrm{mm}$ was generally considered as a hypomobility (restricted ROM). We compared both the VAS and ROM values between discectomy and discoplasty groups before the surgeries and as well 12 months after the operations. Further, we compared the VAS and ROM values before and after the surgeries in the discectomy and discoplasty groups. Statistical procedure was performed with paired and two sample $\mathrm{t}$-tests. The level of significance was considered if $\mathrm{p}<0.05$.

\section{Surgical techniques}

The open joint surgeries were performed under general anesthesia in the Department of Oral \& Maxillofacial Surgery, Stomatologická klinika, Charles University in Prague, Faculty of Medicine in Hradec Králové and the Department of Oral \& Maxillofacial Surgery, Nemocnice Liberec in Liberec, Czech Republic. Antibiotic coverage was given preoperatively (a single dose of clindamycin $600 \mathrm{mg}$ intravenously). Each joint was approached via preauricular incision, followed by blunt dissection to the temporal fascia, which was then incised. The articular capsule was entered through a horizontal incision made just below the zygomatic process and extended anteriorly to the eminence. After exposure of the superior joint space, the capsule was dissected from the lateral border of the disc. The lower joint space was exposed through a horizontal incision in the collateral ligament rim of the disc. The disc was checked for its position and possibility to reposition and carefully freed from its displaced position. In all cases, the displaced disc was found to be located anteriorly and medially. Disc displacement without reduction was defined as a disc position in which the posterior band resided anterior to the condyle in the jaw-closed position and remained anterior to the condyle with opening. The discoplasty procedure was intro- 


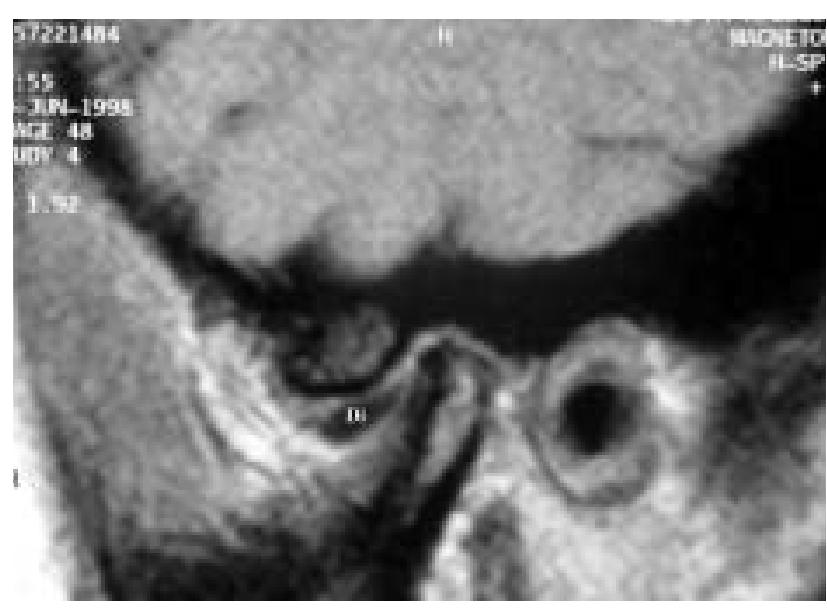

Fig. 1: MRI of the left TMJ before operation revealed anterior disc dislocation without reduction and degenerative changes of the cranioventral portion of the condyle ( $\mathrm{Di}$ discus).

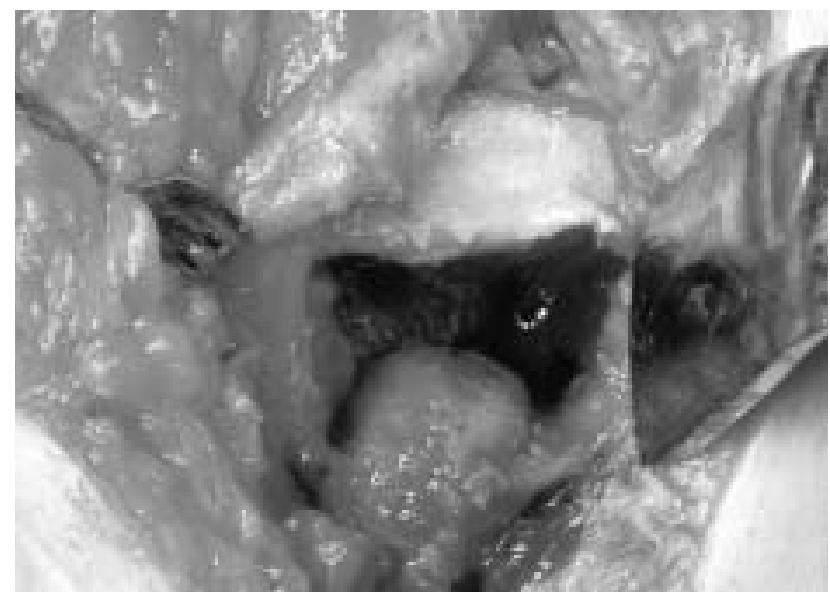

Fig. 3: TMJ after discectomy; all adhesions were removed, the condyle was freed and minimally recontoured.

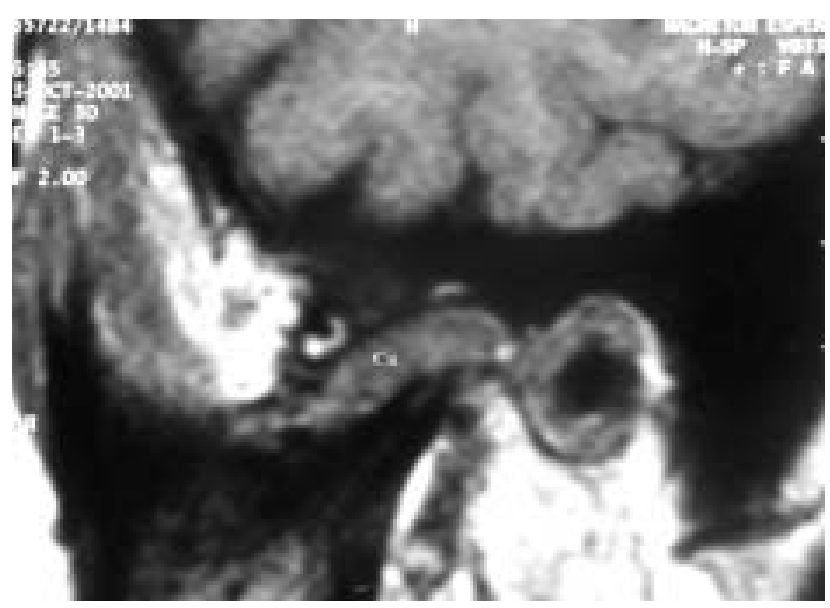

Fig. 2: MRI of the same patient and the same side, 3 years after discectomy and grafting with auricular cartilage $(\mathrm{Ca}-$ cartilage)

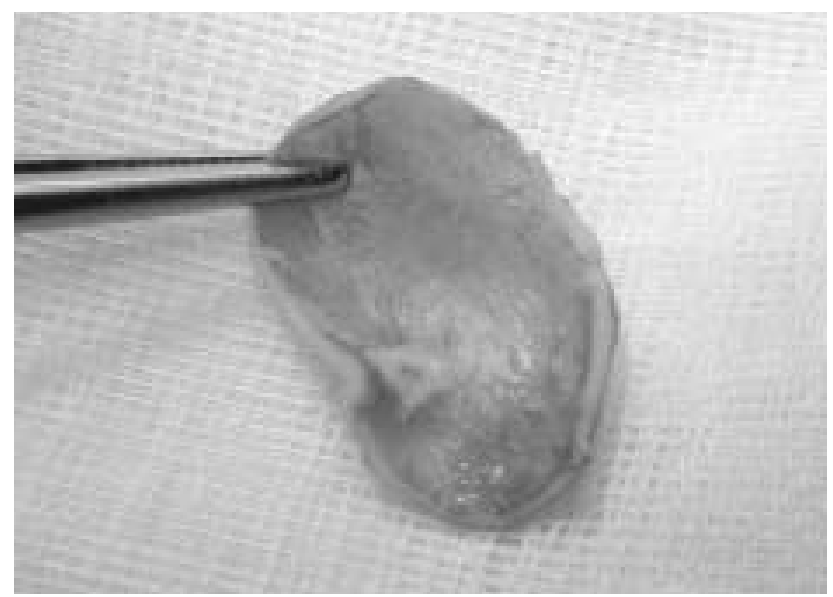

Fig. 4: Sufficient size of the cartilage was harvested from the conchal region with an excellent "boat-like" portion.

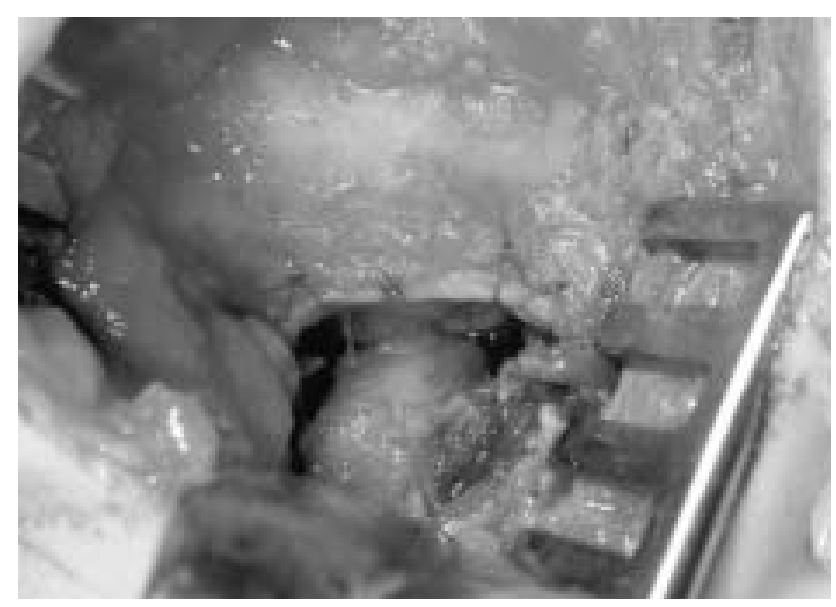

Fig. 5: The cartilage graft was interpositioned in the fossa and secured with three sutures to the capsular remnants. 
duced with a partial wedge-shaped excision of the posterior ligament and the disc was pulled back to localize the distal thickening at position of 12 o'clock on the condyle and interpose the disc between the condylar head and the eminence. When indicated, excessive retrodiscal tissue was excised. The disc was approximatized with four to five slowly absorbable sutures. In addition, an eminectomy was performed in 2 out of 11 TMJs where we performed discoplasty involving vertical bone reduction of about $5 \mathrm{~mm}$ at the apex of the eminence. Four patients underwent a simultaneous condylar shaving. After rinsing the upper and lower joint spaces, we checked the occlusion and movement of the condyle and the disc. The wound was closed in layers. In cases, where the discs were impossible to repair, we removed them to the extent possible (discectomy). The condyle was afterwards freed and mouth opening was checked. During this procedure, there was significant increase of vertical opening. The auricular cartilage graft was harvested from the conchal region and fixed to the fossa in a usual way (15). In five joints, we smoothed condylar irregularities with a diamond bur that allowed the condyle process to be better fitted to the auricular graft (Fig 3,4,5). The wound was then closed in layers. Postsurgical management included combination of pharmacotherapy and physical therapy. Early, sustained postoperative jaw-opening exercise was encouraged. Patients were advised to exercise only within the limits of pleasant tension to avoid possible protective co-contraction of masticatory muscles. Subjects were instructed to eat only soft food in the initial postoperative period and step by step were advanced to harder food as tolerated.

\section{Results}

There were no significant intraoperative complications. We did not meet any infectious complication either, although we used only preoperative dosage of intravenous antibiotic. The harvesting of the cartilage graft as well as the approach to the joint cavity were done through properly scrubbed skin. We did not use any drainage and lavage after the surgery thus the operating field was tightly closed since the sutures were placed. However, on 9 operated sides $(25.0 \%)$ in 8 patients, a transient palsy of the temporal branch of the

Tab. 1: Pain registration before and after surgical treatment for arterior without reduction of the TMJ.

\begin{tabular}{|l|c|c|}
\hline Surgical technique & Preoperative & Postoperative \\
\hline Discoplasty ( $n=11)$ & $\begin{array}{c}63.64 \pm 7.45 \\
(50 \text { to } 70)\end{array}$ & $\begin{array}{c}20.00 \pm 12.45 \\
(10 \text { to } 50)\end{array}$ \\
\hline Discectomy ( $n=25)$ & $\begin{array}{c}70.60 \pm 6.82 \\
(60 \text { to } 80)\end{array}$ & $\begin{array}{c}19.00 \pm 11.64 \\
(0 \text { to } 40)\end{array}$ \\
\hline
\end{tabular}

Pain registered on a 100-mm VAS. Values are mean \pm standard deviation and range (parentheses) facial nerve developed. The VAS scores (mean and standard deviation) at the baseline and 12 months after the surgery are shown in Table 1. The ROM in mm (mean and standard deviation) before the operations and 1 year later are shown in Table 2.

\section{Discoplasty group}

The discoplasty was performed in eleven patients. Four patients underwent a simultaneous condylar shave and two of them underwent eminectomy. The follow-up examination of the patients after surgery was performed from 18 to 48 months (30.0 \pm 13.1$)$. Before operation, all eleven patients presented their level of pain more than 50 on VAS (VAS 63.64 \pm 7.45 ). After the surgery, during 1-year followup, eight patients reported marked subjective improvement in pain level (lower than 30 on VAS), whereas three patients announced 30 on VAS or more. Hence overall on eight operated sides $(72.7 \%)$, the postoperative pain relief

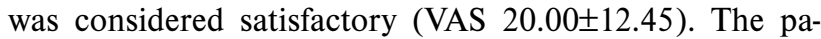
tients who were followed for the discoplasty opened their mouth with a range of 20 to $40 \mathrm{~mm}$ (ROM 33.64 \pm 6.25 $\mathrm{mm}$ ) preoperatively. The maximum of the interincisal distance increased after surgery in all but three patients; however ten patients $(90.9 \%)$ reached $35 \mathrm{~mm}$ or more (ROM $39.09 \pm 2.98 \mathrm{~mm})$. The only patient who opened less $(34$ $\mathrm{mm}$ ), improved ROM after discoplasty from severe limited mouth opening $(20 \mathrm{~mm})$. Four patients reported numbness in the auriculotemporal region and one patient was presented with numbness of her lower lip that was due to mechanical damage to the inferior alveolar nerve caused by the towel clamp used for mandibular traction. On three operated sides, transient palsy of the temporal branch of the facial nerve developed. Normal function of the facial nerve was observed within 4 weeks. No open bite was registered after the surgery.

\section{Discectomy group}

We performed the discectomy on 25 joints in 22 patients (in three subjects bilaterally). In all patients, the autogenous conchal cartilage was used to replace the excised disc. The follow-up examination of the patients after the

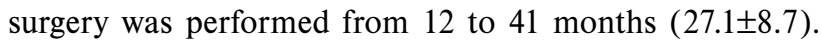
Before the operation, the level of pain of all 22 patients on

Tab. 2: Mouth opening before and after surgical treatment for disc dislocation without reduction of the TMJ.

\begin{tabular}{|l|c|c|}
\hline Surgical technique & Preoperative & Postoperative \\
\hline Discoplasty $(\mathrm{n}=11)$ & $33.64 \pm 6.25$ & $39.09 \pm 2.98$ \\
& $(20$ to 40$)$ & $(34$ to 42$)$ \\
\hline Discectomy $(\mathrm{n}=22)$ & $28.27 \pm 5.36$ & $43.00 \pm 7.08$ \\
& $(20$ to 38$)$ & $(34$ to 58$)$ \\
\hline
\end{tabular}

Mouth opening registered as maximal interincisal distance in $\mathrm{mm}$. Values are mean \pm standard deviation and range (parentheses) 
their 25 operated joints was reported as equal as or higher than 60 on VAS (VAS $70.60 \pm 6.82$ ). On total, on seventeen out of 25 operated sides ( $68 \%$ ), the postoperative pain relief was considered satisfactory (VAS 19.00 \pm 11.64 ). Twentytwo patients who were followed for the discectomy, opened their mouth preoperatively with a range of 20 to $38 \mathrm{~mm}$ (ROM 28.27 $\pm 5.36 \mathrm{~mm}$ ). In all but two patients, the maximum of the interincisal distance increased after surgery. Eighteen patients $(81.8 \%)$ reached postoperatively vertical distance of $35 \mathrm{~mm}$ or more (ROM $43.00 \pm 7.08 \mathrm{~mm}$ ). The other four patients opened their mouth $34 \mathrm{~mm}$ in the followup period. Six patients reported numbness in the auriculotemporal region. On six operated sides, a transient palsy of the temporal branch of the facial nerve developed. Normal function of the facial nerve was observed within 4 weeks in all but one patient. In one case, the paralysis lasted 5 months and was finally also fully rehabilitated. No permanent paresis of the facial nerve was seen. In four patients, we observed temporary open bite on ipsilateral side, which was normalized during the first postoperative week.

\section{Pain}

The minimum pain levels preoperatively were alerting (50 on VAS for discoplasty and 60 for discectomy). After the surgery the VAS values were lower than 30 on 25 sides $(69.4 \%)$. The preoperative VAS values between the discoplasty and discectomy groups were significantly different $(p<.01)$. However, 12 months after the surgery, the VAS values related between the discoplasty and discectomy groups were not statistically different. Comparing the preoperative and postoperative VAS values separately in the discoplasty group itself and similarly in the discectomy group, the paired t-test showed the difference to be significant $(\mathrm{p}<.001)$ for both groups, (Graph 1).

\section{Mouth opening}

Using the cutoff point for maximum of the interincisal opening of more than $35 \mathrm{~mm}$, only 6 patients (16.6\%) ful-

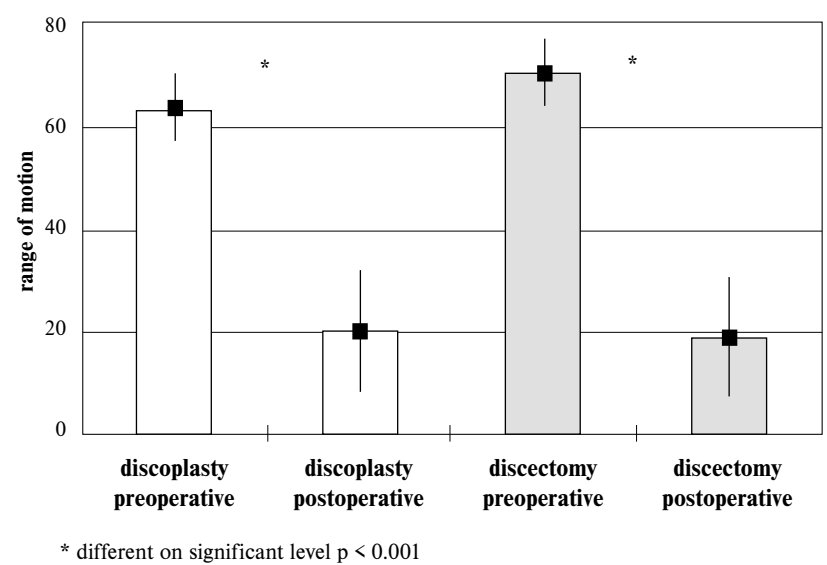

Graph 1: Effect of discoplasty/discectomy on VAS filled the criterion at the baseline. Twelve months after surgery, 31 patients $(86.1 \%)$ were able to open vertically more than $35 \mathrm{~mm}$. Comparing preoperative ROM in patients who underwent discoplasty or discectomy, the distances were significantly different $(p<.05)$. Similar results were seen between groups after the surgeries $(p<.05)$. Relating the preoperative and postoperative interincisal distances in the discoplasty and discectomy groups, the paired $\mathrm{t}$-test showed the difference to be significant ( $p<.05$ for the discoplasty group and $\mathrm{p}<.001$ for the discectomy group), (Graph 2).

\section{Discussion}

Based on the available literature, surgical treatment of TMJ internal derangement has been proved effective for reducing pain and increasing range of motion in about $80 \%$ of patients, regardless of the operating technique (7). The arthroscopy is a good alternative method to open joint surgery, especially if pronounced inflammation of the joint is encountered (12). It has been concluded that arthroscopy should be indicated in disc displacement with or without reduction and in joint luxation in cases of medical treatment failure (4). On the other hand, in patients where internal derangement of the TMJ is confirmed on MRI and subjects suffer from severe intra-articular pain and limited mouth opening, arthroscopy is no longer the treatment of choice. In these cases, open joint surgery has been shown to be more effective (7). The purpose of this study was to evaluate success of the two defined surgical techniques for internal derangement of the TMJ. The study was short-lasting, nonrandomized, and retrospective, with inherent weaknesses of retrospective analysis of data. A VAS used in this study is not a precise method for measuring pain intensity. The ability of some patients to ignore pain because of a higher tolerance limit means that there is a high degree of unreliability in such a scale. The main results of the present study can be summarized as follows:

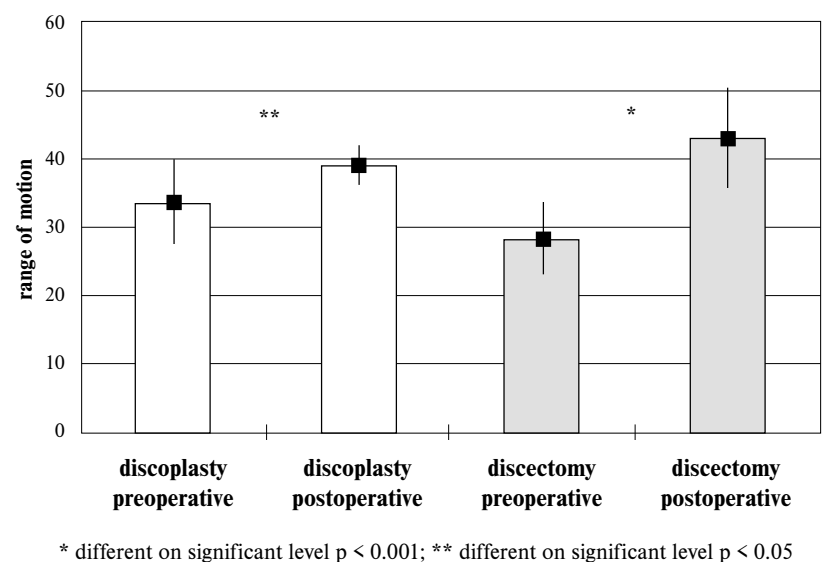

Graph 2: Effect of discoplasty/discectomy on ROM 
1. Patients in both groups subjectively had a satisfactory decrease of pain (VAS $<30)$ : in the discoplasty group $72.7 \%$, in the discectomy group $68.0 \%$, respectively. From that point of view, the discoplasty group had better outcome. However, the difference between both values was not statistically different. The mean VAS value preoperatively was higher in the discectomy group (70.6\%) than in the discoplasty group (63.6\%) and VAS values at 1-year check-up were almost the similar (19.0 for the discectomy group and 20.0 for the discoplasty group).

2. Interincisal distance measured vertically 12 months after the operation increased in all but five patients (three in the discoplasty group and two in the discectomy group). In three of the five subjects, ROM was at the same level as before the surgery. Almost ninety-one percent of the patients $(90.9 \%)$ in the discoplasty group and $81.8 \%$ in the discectomy group, respectively, reached $35 \mathrm{~mm}$ or more of vertical opening. It is necessary to add that the mean ROM before the surgical treatment was lower in the discectomy group $(28.3 \mathrm{~mm})$ than in the discoplasty group $(33.6 \mathrm{~mm})$ and contrary at the 1-year follow-up, the patients operated on by means of discectomy opened on average $43.0 \mathrm{~mm}$ and patients after discoplasty $39.1 \mathrm{~mm}$.

Generally, we tended to use the more aggressive technique in more damaged joints, where discs were more destroyed, and contrary. Hence, we performed the discectomy in patients with more severe signs and symptoms, and the outcomes after the operation were not as excellent as after the discoplasty. So far, we still prefer the discectomy as a method of choice when surgery is necessary. The reason for not advocating the discoplasty is high level of relapse, which necessitates a second operation, usually the discectomy, at a later stage. It is known from a literature that following the disc repositioning, $25 \%$ of patients continued to have disc displacement (13). Discs that have been displaced for a long time have irreversible structural changes, with decreased functional capacity. Experimentally, surgical incision of a disc in animals did not heal (17). However, when our belief in functioning of the disc is strong, no permanent changes are visible, and the repositioning is easy, we decide for the discoplasty.

Generally, only patients suffering from the internal derangement of the TMJ caused by disc dislocation who sustained non-effective intensive conservative treatment are candidates for the operation. In subjects, where the disc is possible to repair and pronounced anatomical disposition as a very steep articular tubercle and/or high and narrow condyle are presented, we consider repositioning followed by reshaping of the tubercle or the condyle. Making the condylar path flat an easy to slide for condyles, we prevent possible failures. There is evidence that immediate and continuous rehabilitation is crucial for obtaining the optimal results after the surgeries. The counseling of the patients about the regular and correct way of exercises to increase range of motion is important, as well.
Applying the modified Criteria for classification of postoperative results (AAOMS) from year 1984, 12 months after surgery 24 patients $(72.7 \%)$ displayed good results, 6 patients $(18.2 \%)$ were evaluated with acceptable results and in 3 cases $(9.1 \%)$ we observed bad results. The majority of the operated patients were without any pain or just with mild pain. Three patients were presented postsurgically with certain level of pain reflecting in above 30 VAS values. Looking at the months beyond 1-year follow-up, the pain was precipitating more extra-articularly and thus it was probably our misdiagnosis of indication for the surgical therapy. Two patients with pain of myofascial origin were educated about isometric exercise and recommended for physiotherapy, behavioral and psychiatric supportive treatment. One patient finally diagnosed as having atypical trigeminal neuralgia was managed with Tegretol (Carbamazepinum, Novartis Pharma AG, Switzerland) after experiencing with no response to Neurontin (Gabapentinum, Parke-Davis GmbH, Germany).

As it is mentioned above, the patients who were operated on more than once were excluded from the study. It is interesting to add, that pain on mandibular movement was the principal reason why other 3 patients after the discoplasty were reoperated. Within 2 months, the patients could not open their mouth more than $28 \mathrm{~mm}$, probably as a consequence of adhesion or fold of the disc. Any opening of the mouth exceeding rotational movement was accompanied with pain. Despite our effort to continue with the physical therapy, patients refused the conservative management and were persuading us to go for another operation. These patients were eventually operated by means of the discectomy and despite persistent certain level of pain evaluating as 50 , 65 , and 35 on VAS respectively, they were satisfied with the result of the second operation. None of these patients was able to open more than $35 \mathrm{~mm}$ and in all of them, we registered facial palsy in the forehead region. In one patient, we registered a permanent damage of the facial nerve. These patients were not in need to be operated on anymore. The joints compromised by previous operations are more likely to develop complication after subsequent surgery. Experience indicates that success of any TMJ surgery after one or more previous surgeries approaches zero (3).

Eriksson and Wesstenson (8) recalled 15 patients on average 29 years after unilateral discectomy and reported to be free of pain and most of them had vertical and excursive movements of mandible at least $35 \mathrm{~mm}$ and $5 \mathrm{~mm}$, respectively. All of the operated joints and most of the unoperated contralateral joints showed condylar flattening and sclerosis radiographically. They suggested that the morphologic changes in the operated joints represented functional adaptative changes. We considered beneficial to use autogenous cartilage to replace the missing disc in all TMJ after discectomies and suggested that the graft could preserve articular surfaces before overloading and subsequent degenerative changes. The first report of using auricular cartilage as 
a disc replacement is attributed to Perko (19). The always appearing question is the fate of the cartilage. Matukas and Lachner (16), during the reoperation, found normal-appearing cartilage, and histological evaluation showed viable hyaline cartilage. The auricular cartilage is a readily available autogenous tissue within the same operative field as the recipient site that can be obtained quickly, and which leaves no donor site deformity. It is not known whether interpositional auricular cartilage grafting provides greater pain relief than discectomy alone (22). In our series, no pain and no deformities were seen at the donor sites in the immediate postoperative period and as well later except one patient with some irregularity of the antehelical rim which was recontoured in the local anesthesia one month after operation.

\section{Conclusions}

Surgical treatment remains the last modality in the cascade of treatments for the internal derangement of the TMJ. If patients have significant mechanical problems such as limited mouth opening, intermittent locking or harsh clicking associated with significant deviations in movement of the TMJ, an open surgery should be considered (6). Plication and other repair procedures are probably adequate if the disc is anteromedially displaced, reducible, and not deformed. For those joints in which the disc is fibrotic, distorted or worn, or simply unhealthy-appearing, discectomy is the indicated procedure. In addition, the eminectomy or condylar shave is recommended in situation where anatomic situation is far to be easy functioning. In all situations where discectomy is performed, the replacement of the disc with autogenous material is indicated. Although only shortterm evaluation is presented, our findings show that both discoplasty and discectomy are effective methods for surgical treatment of the patients suffering from severe temporomandibular pain and limited mouth opening. Discoplasty should be reserved only for patients with not severe changes of the disc.

\section{References}

1. Agerberg G, Lundberg M. Changes in the temporomandibular joint after surgica treatment. A radiologic follow-up study. Oral Surg Oral Med Oral Pathol 1971; 32:865-75.

2. Bakke M, Möller E. Craniomandibular disorders and masticatory muscle function Scand J Dent Res 1992;100:32-8.

3. Bradrick JP, Indresano AT. Failure rates of repetitive temporomandibular joint surgical procedures. J Oral Maxillofac Surg 1992;(suppl 3);50:145-9.

4. Chossegros C, Cheynet F, Gola R, Pauzie F, Arnaud R, Blanc JL. Clinical results of therapeutic temporomandibular joint arthroscopy: A prospective study of 34 arthroscopies with prediscal section and retrodiscal coagulation. $\mathrm{Br} \mathrm{J}$ Oral Maxillofac Surg 1996;34:504-7.

5. Dolwick MF, Reid R, Sanders B, et al. Criteria for TMJ Meniscus Surgery Chicago, IL, American Association of Oral and Maxillofacial Surgeons. 1984:31.
6. Dolwick MF. Disc preservation surgery for the treatment of internal derangements of the temporomandibular joint. J Oral Maxillofac Surg 2001;59:1047-50.

7. Dolwick MF. The role of temporomandibular joint surgery in treatment of patients with internal derangement. Oral Surg Oral Med Oral Pathol Oral Radiol Oral Endod 1997;83:150-5.

8. Eriksson L, Westesson P-L. Discectomy as an effective treatment for painful temporomandibular joint internal derangement: A 5-year clinical and radiolographic follow-up. J Oral Maxillofac Surg 2001;59:750-8.

9. Eriksson L, Westesson PL. Temporomandibular joint diskectomy. No positive effect of temporary silicone implant in 5-year follow-up. Oral Surg Oral Med Oral Pathol 1992;74:259-72.

10. Greene CS, Karbach JJ. Epidemiologic studies of mandibular dysfunction: a critical review. J Prosthet Dent 1982;48:184-90.

11. Gundlach KK. Long-term results following surgical treatment of internal derangement of the temporomandibular joint. J Cranio Max Fac Surg 1990;18:206-9.

12. Holmlund AB, Gynther G, Axelsson S. Diskectomy in treatment of internal derangement of the temporomandibular joint. Follow-up at 1,3, and 5 years. Oral Surg Oral Med Oral Pathol 1993;76:266-71.

13. Kaplan P, Reiskin A, Tu H. Temporomandibular joint arthrography following surgical treatment of internal derangements. Radiology 1987;163:217-20.

14. Kirk WS. Risk factors and initial surgical failures of TMJ arthrotomy and arthroplasty: a four to nine year evaluation of 303 surgical procedures. J Craniomandib Pract 1998; 16:154-61.

15. Krug J, Jirousek Z. Auricular cartilage implantation after discectomy of the temporomandibular joint: a report of 27 cases and review of the literature. J Craniomandib Pract 2003, in press

16. Matukas VJ, Lachner J. The use of autologous auricular cartilage for temporomandibular joint disc replacement: A preliminary report. J Oral Maxillofac Surg 1990;48:348-53.

17. Merrill RG. Historical perspectives and comparisons of TMJ surgery for internal disc derangements and arthroplasty. J Craniomandib Pract 1986;4:74-85.

18. Myrhaug H. A surgical technique for treatment of habitual luxation of the temporomandibular joint (Application in an instance of indurated temporomandibular joint luxation). Quintessence Int. 1972;3:9-12.

19. Perko M. Indikationen und Kontraindikationen für chirurgische Eingriffe am Kiefergelenk. Schweiz Monatsschr Zahnheilkd 1973;83:73-81.

20. Pincock JL, Dann JJ. Auricular cartilage grafting after discectomy of the temporomandibular joint. J Oral Maxillofac Surg 1993;51:256-9.

21. Salonen L, Hellden L, Carlsson GE. Prevalence of signs and symptoms of dysfunction in the masticatory system. J Craniomand Disord Facial Oral Pain 1990; 4:241-50.

22. Sandler NA, Macmillan C, Buckley MJ, Barnes L. Histologic and histochemica changes in failed auricular cartilage grafts used for a temporomandibular joint disc replacement: A report of three cases and review of the literature. J Oral Maxillofac Surg 1997;55:1014-9.

23. Schellhas KP. Internal derangements of the temporomandibular joint: Radiologic staging with clinical, surgical and pathologic correlation. Magn Reson Imaging 1989;7:495-515.

24. Silver CM. Long term results of meniscectomy of the temporomandibular joint J Craniomandib Pract 1985;3:46-57.

25. Walker R, Kalamchi S. A surgical technique for management of internal derangement of the temporomandibular joint. J Oral Maxillofac Surg 1987;45: 299-305.

Submitted July 2003.

Accepted November 2003.

MUDr. et MUDr. Jiři Krug, Ph.D., Charles University in Prague, Faculty of Medicine in Hradec Králové, Department of Dentistry, 50005 Hradec Králové, Czech Republic. e-mail: jirikrug@hotmail.com 